HIGH ENERGY ASTROPHYSICS AND COSMOLOGY

\author{
L. Woltjer \\ European Southern Observatory \\ c/o CERN, CH-1211 Geneva 23, Switserland.
}

\title{
Abstract
}

A brief review is given of cosmological tests based on quasars, of source evolution and of the X-ray background. The cosmological tests are still inconclusive because of the limited material available and the possibility of serious selection effects. Quasars and other extragalactic sources account for most or all of the background in the $1-10$ kev range and very possibly also at higher $X$ - and $\gamma$-ray energies.

High energy astrophysics impacts on cosmology in several ways: quasars or radio galaxies may be used for classical cosmological tests; counts of these objects at different redshifts give information on evolution in the universe; the background at $X-$ and $\gamma$-ray wavelengths contains information on faint sources, hot gas and energetic particles in the universe.

\section{HUBBLE DIAGRAMS FOR QUASARS}

Plots of optical magnitude versus redshift for quasars show much scatter. Selecting appropriately chosen subsamples may lead to some improvement (Setti and Woltjer 1973, Stannard 1973). Studies of the luminosity function at various redshifts (Bahcall and Turner 1978, Turner 1979, Setti and Zamorani 1978) may allow some further mitigation of the effects of the scatter. However, the fundamental uncertainty remains as to whether quasars at high and low redshifts are the same, whether they have the same luminosities or luminosity function. In fact, this might not be particularly probable in view of the large evolution in number densities at fixed luminosity.

A possible method for improving the situation has been developed by Baldwin (1977). Both he and Osmer (1977) have found that the equivalent width of certain emission lines tends to decrease with increasingluminosity, and in principle this could allow a determination of individual absolute magnitudes. 
In a further study, Baldwin et al. (1978) studied the equivalent width of CIV $\lambda 1549$ (W(CIV)) as a function of luminosity in part of a complete sample of flat spectrum radio quasars. The result is reproduced in figure 1 where $W(C I V)$ is plotted against an appropriately defined absolute continuum magnitude at $\lambda_{o}=1550 \AA$. These absolute magnitudes have been calculated on the basis of a $q_{0}=1$ cosmology. We have indicated in the figure the changes resulting from a change to $q_{0}=0$, which are mostly minor.

In the same plot we may now include the IUE observations of 3c273 (Boksenberg et al. 1978), where the cosmological effects relative to the larger redshift quasars are larger. With the present data it is seen that the $\mathrm{fit}$ to the other points is no worse for $q_{0}=0$ than for $q_{0}=1$. On the basis of a similar plot for $W($ Ly $\alpha$ ) Davidson et a1. (1977) concluded that the $q_{o}=1$ fit was substantially better. While at present it may be premature to come to conclusions, the method would seem to be rather powerful, provided selection effects have not influenced figure 1. Also of course we cannot exclude the possibility that the $W(C I V)-L$ relation depends on redshift, although this is perhaps not so likely.

In principle, the absence of points in the upper left part of figure 1 is not surprising since weak lines in faint objects would easily go undetected. In the identifications of the $S 2$ survey (Schaffer 1978) which is partly the basis for figure 1 , there are among the objects with $M \leqslant 1833$ quasars and 5 objects with continuous spectra. Since the relation between $W(\mathrm{CIV})$ and $m$ in figure 1 depends effectively on 3 points only, it is a cause for concern that several points might have to be added further to the left. In addition, Seyfert galaxies like NGC 4151 are far above the mean relation (Boksenberg et al. 1978). As a consequence, if BL Lacs, Seyferts and Quasars are to be considered as one class of objects, the significance of the apparent relation in figure 1 becomes doubtfu1.

Further progress in this area will depend on additional uv data for low redshift quasars and possibly from the inclusion of $X$-ray results.

\section{SOURCE COUNTS AND EVOLUTION}

From the observed number of sources in various redshift intervals in principle the luminosity function $f(L, z)$ may be obtained in different wavelength ranges. Much has been learned about the luminosity functions of radio sources and optical quasars, and data on the X-ray luminosity function should be forthcoming in the near future. While the general evolutionary picture with more sources of high luminosity in the past has been clear for some time, newer data lead to the conclusion that for the radio galaxies large evolutionary changes have occurred at quite 


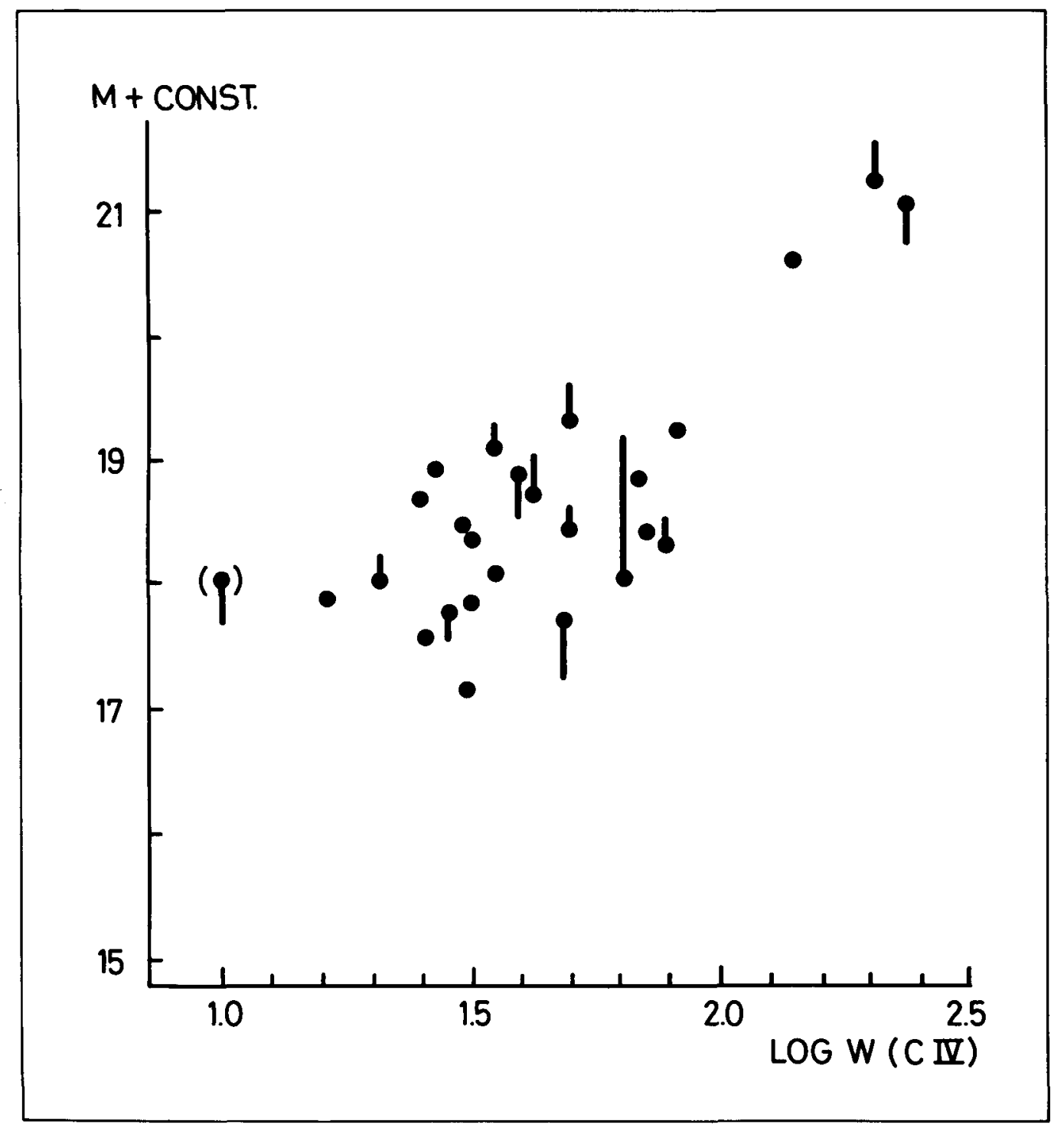

Figure 1: The data of Baldwin et a1. (1978) concerning the equivalent width of the CIV $\lambda 1549$ line versus the absolute magnitude of quasars. The data points are for a $q_{0}=1$ cosmology. The lines indicate the changes if $a \mathrm{q}_{\mathrm{o}}=0$ cosmology is adopted (and the normalization constant in the absolute magnitudes changed in such a way that no change occurs at $z=1.7)$. Changes of less than $0 m 2$ have not been indicated. The data point with the greatest dependence on $q_{o}$ corresponds to $3 C 273$ and is based on IUE data. 
modest redshifts. In fact, Van der Laan, Katgert and de Ruiter (1979) find that there is little evolution of the radio galaxy population between $z=0$ and $z=0.25$ and a very strong one between $z=0.25$ and $z=0.5$. In the case of quasars, the strong evolution seems to occur at higher redshifts, and one might perhaps wonder to what extent this simply means that the nuclei of galaxies become more active at larger $z$, transforming radio galaxies into quasars. Another striking difference pertains to the evolutionary characteristics of the BL Lac objects. The $\log N-\log \mathrm{S}$ relation for BL Lac is much flatter than that for quasars, presumably indicating a rather limited evolution (Setti and Woltjer 1977, Setti 1978). Possibly the BL Lacs are the last evolutionary phase, old quasars which have lost their gas.

The advantage of $X-$ ray studies in the analysis of source counts is that whereas it is frequently difficult to isolate optically proper samples of quasars and Seyferts in the midst of all other stars and galaxies, such objects seem to be the dominant constituent of the X-ray sky.

\section{THE X-RAY BACKGROUND}

The diffuse $X-$ and $\gamma$-ray background in principle consists of two components: unresolved faint discrete sources and a truly diffuse component. It is as yet unclear if the latter makes a significant contribution.

At energies in the 1 - $10 \mathrm{kev}$ range much information has become available about various classes of sources. The main contributors appear to be the following:

1. Clusters of galaxies

2. Normal galaxies

3. Seyferts

$$
\begin{aligned}
& 14 \% \text { (Schwarz 1978) } \\
& 1-6 \% \\
& \text { f } 6 \% \text { (E1vis et al. 1978) } \\
& \{>9 \% \text { (Véron 1979) } \\
& \text { 4. Quasars }>70 \% \quad \text { (Setti and Woltjer 1979) }
\end{aligned}
$$

5. Other active galaxies

1. Clusters of galaxies: With the discovery of many clusters earlier estimates have had to be increased substantially. The percentage listed is on the assumption of no evolution.

2. Normal galaxies: Setti and Woltjer (1970) estimated a $1.2 \%$ contribution assuming the same ratio of optical to X-ray luminosity for our galaxy and other galaxies and assuming no evolution. With massive binaries and supernova remnants being major contributors, the flux of a galaxy should be proportional to the rate of star formation. The situation is the same as that considered by Lichti et al. (1977) in connection with the gamma ray background. From the models considered by them it appears that various evolutionary scenarios increase the predicted background by a factor of $2-5$. 


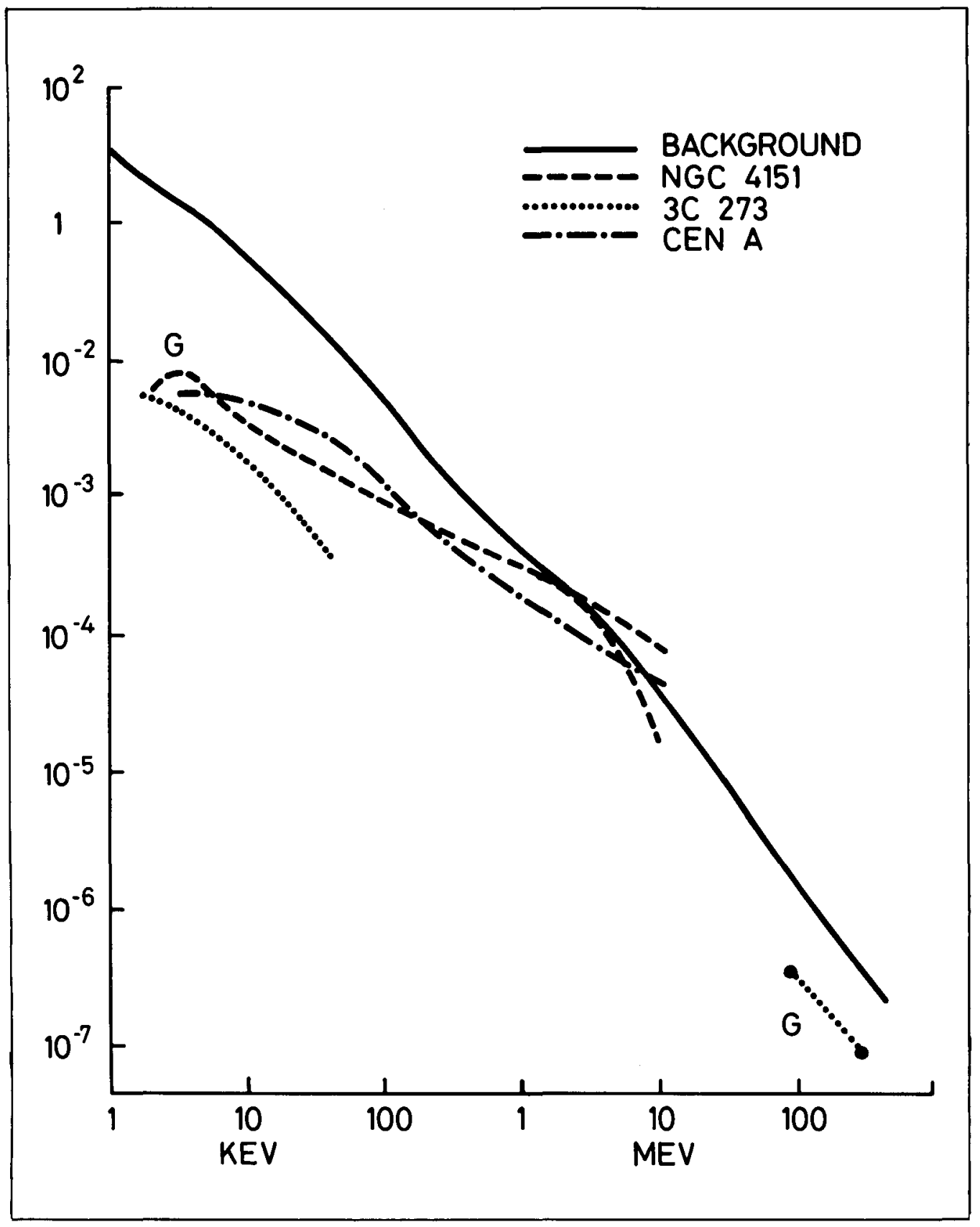

Figure 2: The spectrum of the X-ray background, the Seyfert galaxy NGC 4151 (the high value at a few Mev from Di Cocco et a1. 1977, and the low and probably best value from Schönfelder 1978), the quasar 3C273 and the active galaxy Cen $A$. Two data points on our own galaxy are labelled G. The normalization of the vertical flux scale for different objects is arbitrary. 
3. Seyferts: Elvis et a1. (1978) constructed an X-ray luminosity function for Seyfert galaxies on the basis of Uhuru and Ariel data. Without evolution, they find that Seyferts with $L_{x}>10^{42}$ ergs/sec contribute $6 \%$ of the background. Véron (1979) has constructed an optical luminosity function for Seyfert nuclei and with an assumed average ratio $\mathrm{L}_{\mathrm{x}} / \mathrm{L}_{\text {opt }}$ finds a contribution of $9 \%$ (from objects with $\mathrm{L}_{\mathrm{x}}>10^{42}$ ergs/sec); However, the Véron luminosity function extends to lower luminosities without cutoffs; speculatively extending it down to $\mathrm{M}_{\mathrm{v}}=-17$ he obtains a large part of the total background, even without evolution.

4. Quasars: On the basis of four quasars, Setti and Woltjer (1979) estimated a mean ratio of $\mathrm{L}_{\mathrm{x}} / \mathrm{L}_{\mathrm{opt}}$ similar to that for the more luminous $X$-ray Seyfert nuclei. Combined with the observed optical counts of quasars, $70 \%$ of the background is obtained from quasars with $\mathrm{B}<20$. Several conclusions follow; in particular, a steep extension to the quasar counts to fainter magnitudes (1ike considered by Bohuski and Weedman 1979) would lead to an excessive background. The same would be the result of a picture in which quasars are "local", with the redshift due to non cosmological effects. Recent HEAO-2 data on larger numbers of quasars seem to confirm these conclusions. BL Lac objects seem to have $\mathrm{L}_{\mathrm{x}} / \mathrm{L}_{\text {opt }}$ ratios comparable to those for quasars. However, as the slope of their $\log \mathrm{N}-\log \mathrm{S}$ relation is less steep than for quasars, their contribution to the background is less important.

5.0ther active galaxies: Some radio galaxies (like Cen A) have been observed as X-ray sources as well as some other active galaxies. The total contribution of such objects to the background remains uncertain.

From these results it is apparent that much and possibly a11 of the $1-10 \mathrm{kev}$ background is due to unresolved sources. There is no evidence for a truly diffuse component in that energy range.

A satisfactory theory of the $X-$ and $\gamma$-ray background should explain not only the flux at one energy, but the whole spectrum. The present data appear to be inadequate to do this in a detailed way, but some broad features may be noted (see figure 2).

(a) Clusters of galaxies appear to be thermal emitters with typical temperatures around $7 \mathrm{kev}$ (Mushotzky et al. 1978). At higher energies their contribution should be negligible.

(b) Estimates of the total emission from out Galaxy in the $1-10$ kev range and around $100 \mathrm{Mev}$ suggest that the average spectrum is not very different (possibly slightly flatter) from that of the background.

(c) The spectrum of NGC 4151 (Di Cocco et al. 1977, Schönfelder 1978) is definitely flatter than that of the background. As a consequence, it is no surprise that Schönfelder could obtain the 1 - 10 Mev background with Seyferts alone. 
(d) The spectrum of $3 \mathrm{C} 27.3$ is known with modest accuracy in the 1 - $100 \mathrm{kev}$ range. Variability seriously limits the possiblity of combining data from different experiments. A high latitude $100 \mathrm{Mev}$ source seen by Cos $B$ has been tentatively identified with 3 C 273 (Swanenburg et al. 1978). If this identification is correct, the average spectrum comes out flatter than that of the background. Quasars are therefore likely to make a major contribution also at higher energies.

(e) X-ray data on Cen A (Worral et al. 1979, Hall et a1. 1976) suggest a spectrum flatter than that of the background. Again strong variability is found. Assuming the same radio to $\mathrm{X}$-ray flux for all radio galaxies, Strong et al. (1976) could obtain the whole background at a few Mev from such objects.

In the present stage of our knowledge it would seem then that normal galaxies make a modest contribution to the background at most energies and clusters of galaxies at low energies. Quasars, Seyferts and other active galaxies probably account for the larger part at all energies (figure 3 ). In fact, the flatness of the spectra of 3C273 and NGC 4151 shows that most of the Seyferts and Quasars should have steeper spectra or cutoffs in the $10-1000 \mathrm{kev}$ range if the background is not to be exceeded. The spectrum of the background may largely reflect the distribution over frequency of these cutoffs.

There is at the moment no evidence for any extragalactic diffuse background. As a result, the dozen or so theories for the diffuse background have only upper limits to go by. In particular, there is no evidence as yet for a hot intergalactic gas. The observations of $\mathrm{X}$-ray emission from superclusters (Murray et al. 1978) may nevertheless indicate that gas is an important constituent of the universe. But inferences about a truly universal intergalactic gas will have to await a more accurate evaluation of the contribution of sources in the $1-100 \mathrm{kev}$ range.

Having found that sources contribute much of the background, measurements of the background distribution give some information about the source distribution as we11. The background in the $8-38 \mathrm{kev}$ range is isotropic on a large scale $(\cos \theta)$ to within $2 \%$ (peak to peak), while for part of the sky in $20^{\circ} \times 20^{\circ}$ bins the variations are less than $+4 \%$ (Schwartz 1970). Since the quasars seem to account for no less than $50 \%$ of the total, it would follow that their large scale distribution should be isotropic to within $4 \%$ peak to peak and their variation in numbers less than $+8 \%$ in $20^{\circ} \times 20^{\circ}$ regions. Various optical observers have suspected that there are large regional variations in the area densities of quasars. The X-ray data make this improbable. Instead one may suspect that the steep $\log \mathrm{N}$ - los $\mathrm{S}$ relation for quasars combined with uncertain limiting magnitudes and possibly some interstellar absorption is responsible for these variations in area density. 


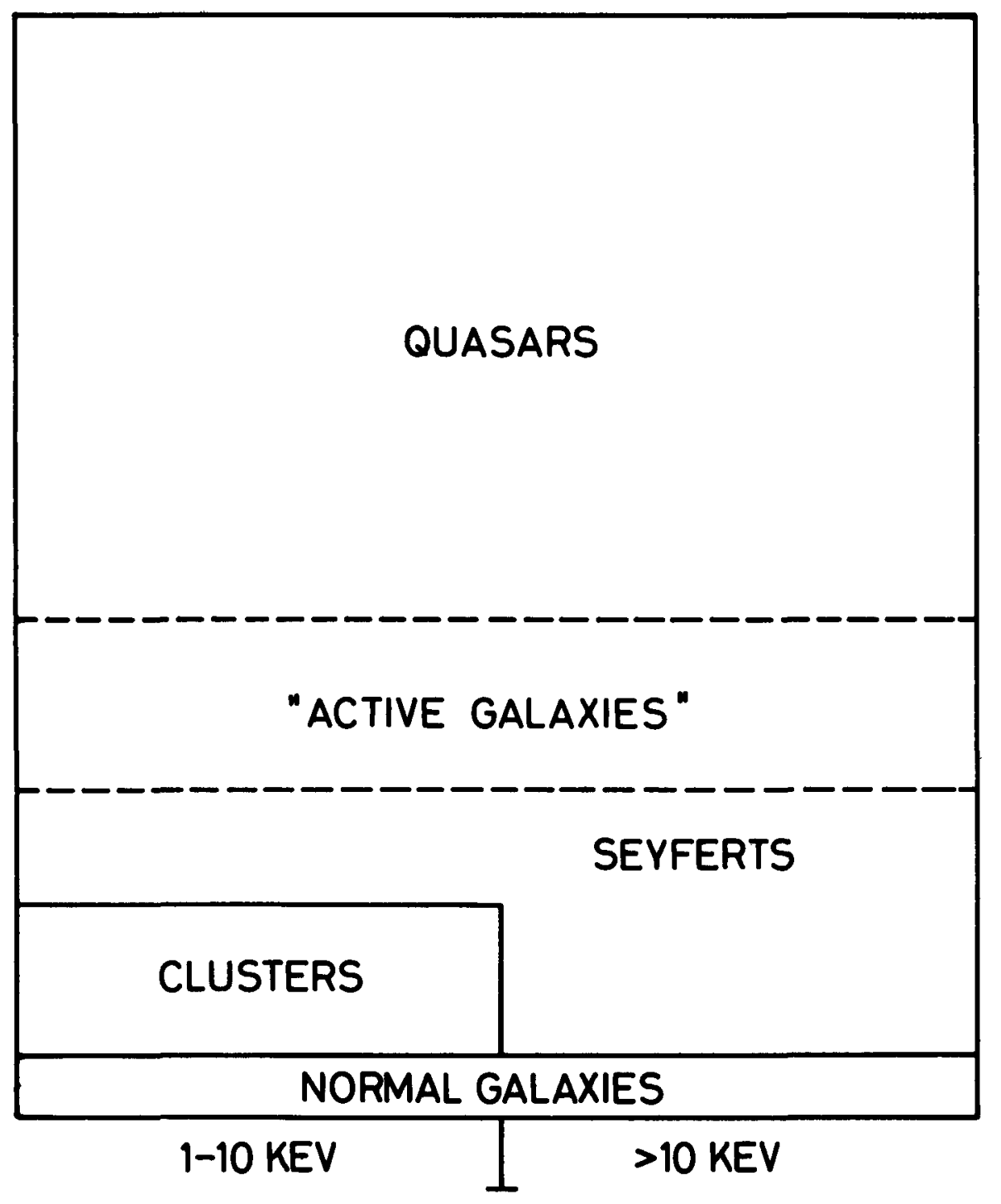

Figure 3: Schematic estimates of the relative contribution of various types of sources to the X-ray background at low and at high energies. The latter are still extremely uncertain. 


\section{REFERENCES}

Bahcal1, J.N. and Turner, E.L.: 1978, Physica Scripta 17, 353.

Baldwin, J.A.: 1977, Ap.J. 214, 679.

Baldwin, J.A., Burke, W.L., Gaske11, C.M. and Wampler, E.J.: 1978, Nature 273, 431 .

Bohuski, T.J. and Weedman, D.W.: 1979, Ap.J. 231, 653.

Boksenberg, A. et al.: 1978, Nature 275, 404.

Davidsen, A.F., Hartig, G.F. and Fastie, W.G.: 1977, Nature 269, 203.

Di Cocco, G. et a1.: 1977, Nature 270, 319.

Elvis, M., Maccacaro, T., Wilson, A.S., Ward, M.J., Penston, M.V., Fosbury, R.A. and Perola, G.C.: 1978, M.N.R.A.S. 183, 129.

Ha11, R.D., Meegan, C.A., Walraven, G.D., Djuth, F.T. and Haymes, R.C.: 1976, Ap.J. 210, 631.

Kinzler, R.L., Johnson, W.N. and Kurfess, J.D.: 1978, Ap.J. 222, 370.

Lichti, G.G., Bignami, G.F. and Pau1, J.A.: 1977, ESA SP-124, 207.

Murray, S.S., Forman, W., Jones, C. and Giacconi, R.: 1978, Ap.J.Lett. $219, \mathrm{~L} 89$.

Mushotzky, R.F., Serlemitsos, P.J., Smith, B.W., Boldt, E.A. and Holt, S.S.: 1978, Ap.J. 225, 21 .

Osmer, P.S.: 1977, Ap.J. 214, 1.

Schönfelder, V.: 1978, Nature $274,344$.

Schwartz, D.A.: 1970, Ap.J. 162, 439.

1978, Ap.J. 220, 8.

Setti, G.: 1978, Pittsburgh Conference on BL Lac Objects, Pittsburgh U., ed. A.M. Wolfe, p. 385.

Setti, G. and Woltjer, L.: 1970, Astroph. \& Space Sci. 9, 185.

1973, Ap.J.Lett. 181, L61.

1977, Ap.J.Lett. 218, L33.

1979, Astron. Astrophys. 76, L1.

Setti, G. and Zamorani, G.: 1978, Astron. Astrophys. 66, 249.

Shaffer, D.B.: 1978, A.J. 83, 209.

Stannard, D.: 1973, Nature 236, 295.

Strong, A.W., Wolfendale, A.W. and Worra11, D.M.: 1976, M.N.R.A.S. 175, $23 \mathrm{P}$.

Swanenburg, B.N. et a1.: 1978, Nature 275, 298.

Turner, E.L.: 1979, Ap.J. 231, 645.

Van der Laan, H., Katgert, P. and de Ruiter, H.: 1979, in "Astronomica1 Uses of the Space Telescope" (ESA/ESO), p. 301.

Véron, P.: 1979, Astron. Astrophys., to be published.

Worral, D.M., Mushotzky, R.F., Boldt, E.A., Holt, S.S. and Serlemitsos, P.J.: 1979, NASA TM 30261 . 